\title{
Implicit Large Eddy Simulations for NACA0012 Airfoils Using Compressible and Incompressible Discontinuous Galerkin Solvers
}

\author{
Esteban Ferrer, Juan Manzanero, Andres M. Rueda-Ramirez, \\ Gonzalo Rubio, and Eusebio Valero
}

\section{Introduction}

High order Discontinuous Galerkin (DG) methods provide accurate solutions by enabling arbitrarily high polynomial approximations inside each grid element. For high order polynomials, the numerical errors are not distributed along all wavenumbers but localised at high wave-numbers [1-5]. This characteristic of high order methods results in very accurate simulations with low dissipative and dispersive errors. Although this characteristic seems a-priori beneficial for well resolved simulations, when computing under-resolved Large Eddy Simulations (LES), it can prove difficult to obtain stable simulations. In implicit (or under-resolved) Large Eddy Simulations (iLES), the smallest numerical eddies are larger than would have been in a finer mesh, leading to numerical under-resolution (i.e. coarse grid or low polynomial order) and aliasing [6]. Various methods have been proposed to stabilise under-resolved computations with aliasing. Among others, split forms or skew symmetric variants [7, 8]), localised interior penalty fluxes [9], over-integration [1012] or filtering [13] may be incorporated into the solver to stabilize the computations and remove or alleviate the aliasing.

Contrarily to low order methods, high order methods do not have enough inherent numerical dissipation in under-resolved simulations, to dissipate large flow structures (when compared to Kolmogorov scales). Therefore, computation of iLES flows using high order DG solvers require localised dissipative mechanisms to dissipate flow structures close to cut-off size. In what follows, we compare two

\footnotetext{
E. Ferrer $(\varangle) \cdot$ J. Manzanero $\cdot$ A. M. Rueda-Ramirez $\cdot$ G. Rubio $\cdot$ E. Valero ETSIAE-UPM (School of Aeronautics - Universidad Politécnica de Madrid), Madrid, Spain

CCS-UPM (Centre for Computational Simulation - Universidad Politécnica de Madrid), Madrid, Spain

e-mail: esteban.ferrer@upm.es
} 
dissipative stabilising mechanisms that enable the simulation of turbulent underresolved flows. On the one hand, we use a compressible formulation with an energy conserving split-form and dissipation through Roe fluxes [14]. On the other hand, the incompressible solver uses the viscous discretisation through interior penalty formulation to enhance stability [9]. We challenge both formulations with a NACA0012 airfoil at various angles of attack in turbulent regimes, to explore both accuracy and stability. We compare simulated results to experimental data and simulations using low order methods (Xfoil and Ansys-Fluent).

\section{Methodologies}

We first introduce the two different mechanisms used to stabilise both compressible and incompressible high order DG formulations. The explanation included here is brief and aims only at introducing the fundamental concepts and motivating ideas. Further details can be found in the following references by the authors [9, 14].

The 3D Navier-Stokes equations can be written as:

$$
\mathbf{u}_{t}+\nabla \cdot \boldsymbol{F}_{e}=\nabla \cdot \boldsymbol{F}_{v},
$$

where $\mathbf{u}$ is the vector of conservative variables $\boldsymbol{u}=\left(\rho, \rho v_{1}, \rho v_{2}, \rho v_{3}, \rho e\right)^{T}$ in compressible solvers. For incompressible solvers $\boldsymbol{u}=\left(v_{1}, v_{2}, v_{3}\right)^{T}$ and Eq. (1) is complemented with $\nabla \cdot \boldsymbol{u}$. Details on the definition of inviscid and viscous solvers can be found in $[9,14]$. To derive discontinuous Galerkin schemes, we consider Eq. (1) for one mesh element $e l$, multiply by a locally smooth test function $\phi_{j}$, for $0 \leq j \leq P$, where $P$ is the polynomial degree, and integrate on $e l$ :

$$
\int_{e l} \mathbf{u}_{t} \phi_{j}+\int_{e l} \nabla \cdot \boldsymbol{F}_{e} \phi_{j}=\int_{e l} \nabla \cdot \boldsymbol{F}_{v} \phi_{j}
$$

We can now integrate by parts the inviscid fluxes, $\boldsymbol{F}_{e}$, integral to obtain a local weak form of the equations (one per mesh element):

$$
\int_{e l} \mathbf{u}_{t} \phi_{j}+\int_{\partial e l} \boldsymbol{F}_{e} \cdot \mathbf{n} \phi_{j}-\int_{e l} \boldsymbol{F}_{e} \cdot \nabla \phi_{j}=\int_{e l} \nabla \cdot \boldsymbol{F}_{v} \phi_{j},
$$

where $\mathbf{n}$ is the normal vector at element boundaries $\partial e l$. We replace discontinuous fluxes at inter-element faces by a numerical inviscid flux, $\boldsymbol{F}_{e}^{*}$, to obtain a weak form for the equations for each element,

$$
\int_{e l} \mathbf{u}_{t} \cdot \phi_{j}+\int_{\partial e l} \boldsymbol{F}_{e}^{*} \cdot \mathbf{n} \phi_{j}-\int_{e l} \boldsymbol{F}_{e} \cdot \nabla \phi_{j}=\int_{e l} \nabla \cdot \boldsymbol{F}_{v} \phi_{j},
$$


where, we have omitted the fluxes at external boundaries, for simplicity. This set of equations for each element is coupled through the inviscid fluxes $\boldsymbol{F}_{e}^{*}$ and governs flow behaviour. Note that one can proceed similarly and integrate by parts the viscous terms (see $[9,15]$ ), but here for simplicity we retain the volume integral.

$$
\int_{e l} \mathbf{u}_{t} \cdot \phi_{j}+\int_{\partial e l} \underbrace{\boldsymbol{F}_{e}^{*} \cdot \mathbf{n}}_{\text {Riemann solver }} \phi_{j}-\int_{e l} \boldsymbol{F}_{e} \cdot \nabla \phi_{j}=\int_{e l}(\underbrace{\nabla \cdot \boldsymbol{F}_{v}}_{\text {Viscous term }}) \cdot \phi_{j}
$$

The non-linear inviscid and viscous terms that can be discretised to control dissipation in the numerical scheme have been underlined.

Riemann solvers are the classic option to include numerical dissipation in DG schemes $[16,17]$, since they naturally arise when discretising the non-linear terms. Comparison of different fluxes for homogeneous turbulence can be found in $[14,18]$. A different option is to modify the viscous terms to enhance its dissipative properties. The latter has been proposed in [9] using an increased penalty parameter (compared to the minimum required to ensure coercivity of the scheme) when discretising the viscous terms using a interior penalty formulation.

\subsection{Compressible DGSEM Solver}

The compressible solver uses conservative variables to solve the Navier-Stokes equations. We use a particular nodal variant of DG methods: the Discontinuous Galerkin Spectral Element Method (DGSEM), see for example [19]. In addition, the compressible formulation is modified to be energy preserving [20]. The required split-form necessitate Gauss-Lobatto points to cancel out boundary terms using the summation-by-parts simultaneous-approximation-term property (SBP-SAT). The interested reader is referred to [5, 20-22]. These energy conserving schemes are designed to remain stable and energy conserving and consequently do not necessitate additional localised numerical dissipation. Nonetheless, in this work we introduce dissipation through Roe fluxes, to enhance robustness at high Reynolds numbers. Additionally, viscous terms are discretised using the Bassi-Rebay 1 (BR1) scheme, which is equivalent to the interior penalty formulation when using GaussLobatto points and hexahedral elements [23]. Let us note that this formulation for the viscous fluxes is neutrally stable [24] and adds the minimum dissipation required to achieve a stable scheme, whilst others may introduce some extra dissipation. Other techniques are available to discretise second order derivatives and can be found in the classic review by Arnold et al. [15]. 


\subsection{Incompressible DG-Fourier Solver}

Flow solutions of the incompressible Navier-Stokes equations, are obtained from the 3D unsteady high order $h / p$ Discontinuous Galerkin-Fourier solver [9, 2528]. The solver uses a second order stiffly stable approach to discretise the NS equations in time whilst spatial discretisation is provided by the discontinuous Galerkin-Symmetric Interior Penalty formulation with modal basis functions in the $x-y$ plane. Here, $x$ represents the streamwise flow direction and $y$ is the normal direction. Spatial discretisation in the $z$-direction (here defining the spanwise airfoil length) is provided by a purely spectral method that uses Fourier series and allows computation of spanwise periodic three-dimensional flows. Since high order methods (e.g. discontinuous Galerkin and Fourier) are unable to provide enough numerical dissipation to enable under-resolved high Reynolds computations (e.g. as necessary in Large Eddy Simulations), we have adapted the original laminar version of the solver to increase (controllably) the dissipation and enhance the stability in under-resolved simulations [9]. This dissipative formulation has minimal impact on well resolved flow regions and its implicit treatment does not restrict the use of relatively large time steps, thus providing an efficient stabilization mechanism for Large Eddy Simulations. The solver has been widely validated for a variety of flows, including bluff body flows, airfoil and blade aerodynamics and vertical axis turbines under static and rotating conditions [9, 25-30].

\section{Numerical Results}

This section considers a NACA0012 airfoil at $R e=1 \times 10^{4}, R e=1 \times 10^{5}$ and $R e=1 \times 10^{6}$ (based on the airfoil chord $c$ ) for a range of Angles of Attack $(A o A)$ : $0^{\circ} \leq A o A \leq 10^{\circ}$. In what follows we compare incompressible and compressible simulations using polynomial orders $P=3$ and $P=4$. The averaged values have been computed after the development of three dimensional flow. The compressible solver uses a hexahedral mesh with 18,000 elements, which for $\mathrm{P}=3$ and 4 result in 1.1 and 2.2 million degrees of freedom. The incompressible solver, uses a mixed tri-quad 2D mesh and is expanded using Fourier in the homogeneous third direction (here 16 Fourier modes). Depending on the angle of attack, the resulting meshes include 0.6 to 1 million degrees of freedom. Meshes for the two solvers and for $A o A=0^{\circ}$ are depicted in Fig. 1. Finally, all the simulations are computed with both DG solvers and consider a periodic spanwise lengths of $L_{z} / c=0.1$. Note that we have not observed significant differences in the results when increasing the spanwise length. Statistics are accumulated during at least 40 convective time scales (based on the airfoil chord) and starting after the turbulent flow has developed (typically an initial transient of 10 convective time scales). 


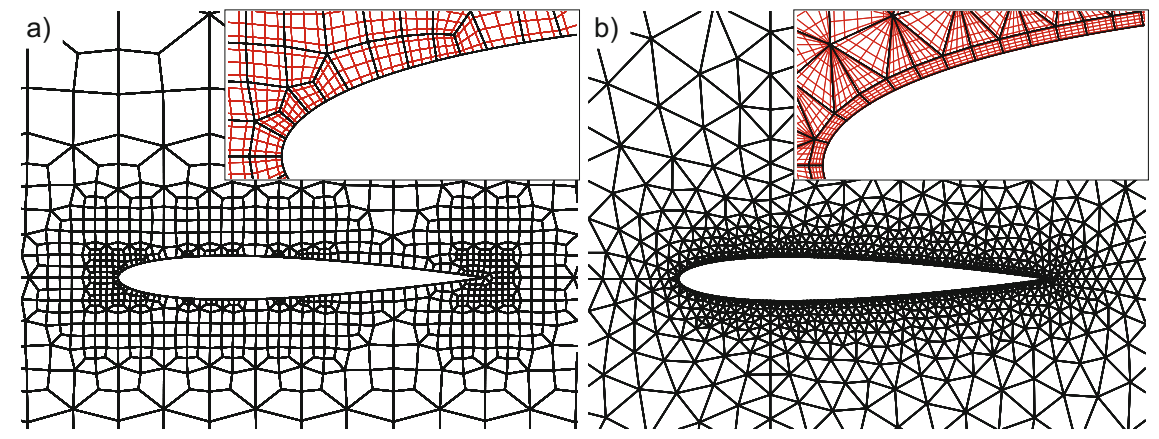

Fig. 1 Meshes for NACA0012 airfoil: (a) Hexahedral mesh for compressible solver and (b) mixed tri-quad mesh for incompressible solver. Inset figures show high order polynomial mesh for order $\mathrm{P}=4$

$\operatorname{Re}=1.000 .000$

$\mathrm{A} \circ \mathrm{A}=0 \mathrm{deg}$

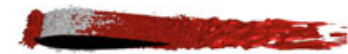

contrours of velocity: $[0.85 ; 1.2]$
$\operatorname{Re}=1.000 .000$

$A \circ A=5 \mathrm{deg}$

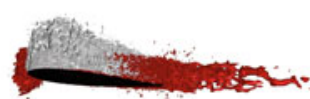

$\mathrm{Re}=1.000 .000$

$A \circ A=10 \mathrm{deg}$

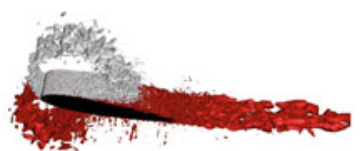

Fig. 2 NACA0012 airfoil at $R e=1 \times 10^{6}$, from left to right: $A o A: 0^{\circ}, A o A: 5^{\circ}$ and $A o A: 10^{\circ}$. Simulations are obtained using the incompressible DG solver

\section{1 $R e=1 \times 10^{6}$ and Various Angles of Attack}

We start by illustrating the highest Reynolds number case, which is the most challenging in terms of stability and robustness. To illustrate the range of the flow behaviour at various $A o A$ s, we show in Fig. 2, velocity contours for $A o A: 0^{\circ}, 5^{\circ}$ and $10^{\circ}$, computed using the incompressible DG solver. It can be seen that at $R e=1 \times 10^{6}$ the flow remains attached for all angles, and that only mild separation is seen near the trailing edge. We will see in the next section that at lower Reynolds numbers this is not necessarily the case.

Figure 3 compares the aerodynamic coefficients with experimental data for various angles of attack and the two solvers. Figure 3 a shows the lift coefficient against the AoA and Fig. $3 \mathrm{~b}$ depicts the Lift-Drag Polar for $R e=1 \times 10^{6}$. We observe very good agreement with experimental data for both solvers. 

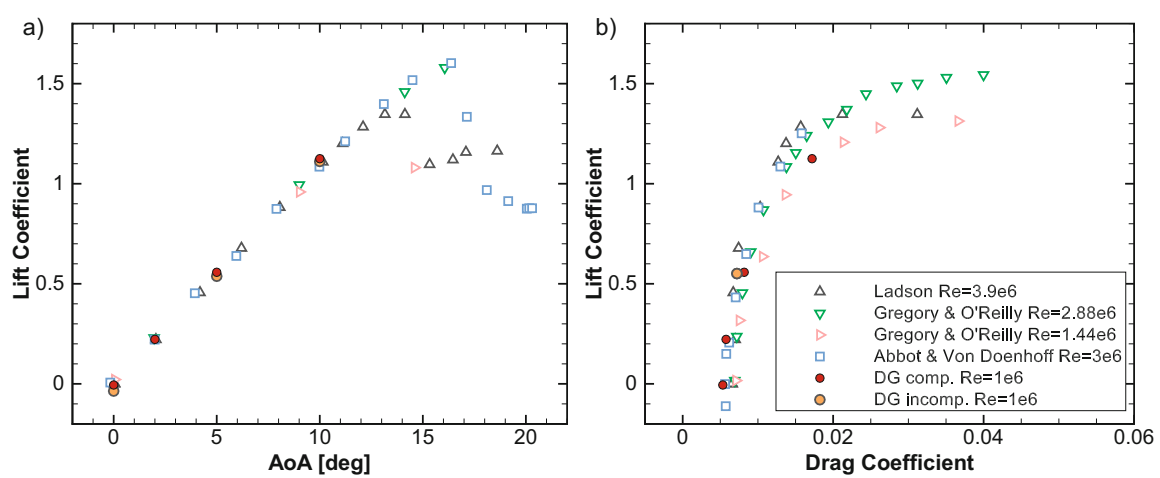

Fig. 3 NACA0012 airfoil at $R e=1 \times 10^{6}$ : (a) Lift coefficient vs angle of attack and (b) LiftDrag Polar. Compressible (comp.) and incompressible (incomp.) DG simulations are compared to experimental data sets of Ladson [31], Gregory and O'Reilly [32], Abbot and Von Doenhoff [33]

\section{2 $A o A=5^{\circ}$ and Various Reynolds Numbers}

Having shown the overall good performance in terms of aerodynamic quantities at the most challenging Reynolds numbers, we now focus our attention on the angle $A o A=5^{\circ}$ and compare the usability of the solvers to study the NACA0012 boundary layer evolution.

First, we compare the aerodynamic coefficients for $A o A=5^{\circ}$, and Reynolds numbers $R e=1 \times 10^{5}$ and $R e=1 \times 10^{6}$, using the incompressible and compressible solvers, both with polynomial order $\mathrm{P}=3$ and $\mathrm{P}=4$, in Table 1 . We observe good agreement for the highest polynomial order. Small discrepancies are attributed to post-processing of statistics and lack of near wall resolution when using $\mathrm{P}=3$, which influences mainly the drag coefficient and particularly viscous drag. For completeness, we depict the flow evolution within the boundary layer using both solvers in Fig. 4. It can be seen that detachment near the trailing edge is similar for both solvers. Regarding transition to turbulence (represented by fluctuations in velocity contour), both solvers capture transition on the suction side. The compressible solver shows a transition location near the maximum thickness

Table 1 NACA0012 airfoil at $A o A=5^{\circ}$ for $\operatorname{Re}=1 \times 10^{5}$ and $\operatorname{Re}=1 \times 10^{6}$

\begin{tabular}{l|l|l|l|l}
\hline & \multicolumn{2}{|l|}{$R e=1 \times 10^{5}$} & \multicolumn{2}{l}{$R e=1 \times 10^{6}$} \\
\cline { 2 - 5 } & $\mathrm{Cl}$ & $\mathrm{Cd}$ & $\mathrm{Cl}$ & $\mathrm{Cd}$ \\
\hline DG comp. $\mathrm{P}=3$ & 0.588 & 0.028 & 0.567 & 0.005 \\
\hline DG comp. $\mathrm{P}=4$ & 0.575 & 0.025 & 0.558 & 0.008 \\
\hline DG incomp. $\mathrm{P}=3$ & 0.484 & 0.028 & 0.538 & 0.017 \\
\hline DG incomp. $\mathrm{P}=4$ & 0.545 & 0.018 & 0.551 & 0.007 \\
\hline
\end{tabular}

Comparison of Lift and Drag using the DG compressible and DG incompressible solvers and two polynomial orders $\mathrm{P}=3$ and $\mathrm{P}=4$ 


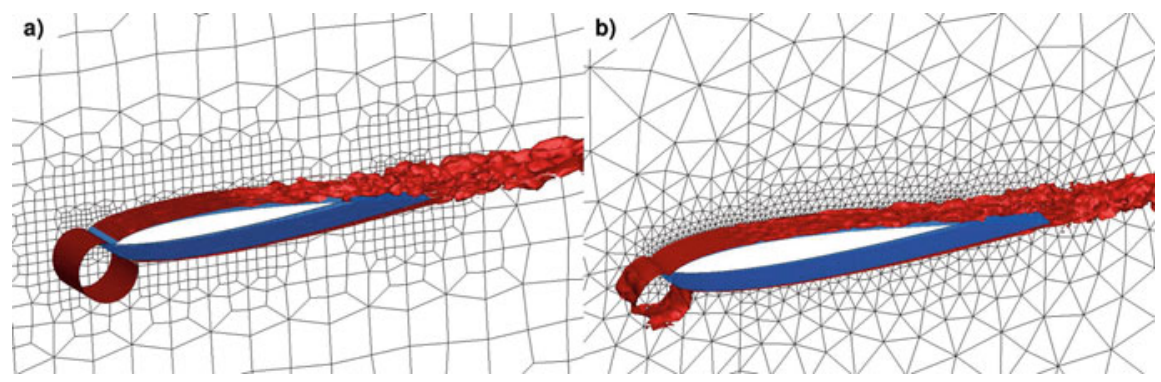

Fig. 4 NACA0012 airfoil at $R e=1 \times 10^{5}$ and $A o A=5^{\circ}$ for $\mathrm{P}=4$ : (a) Compressible DG solver. (b) Incompressible DG solver

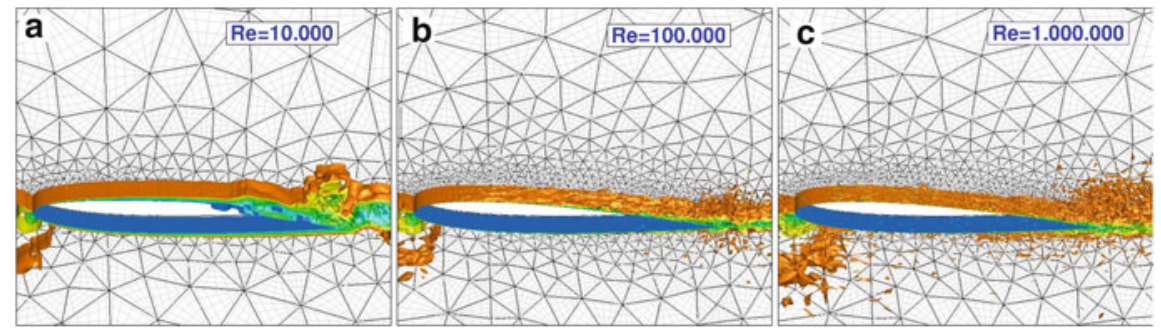

Fig. 5 NACA0012 airfoil at $A o A: 5^{\circ}$ for (a) $R e=1 \times 10^{4}$, (b) $R e=1 \times 10^{5}$ and (c) $R e=1 \times 10^{6}$. Velocity magnitude isocontours and unstructured mesh details are included

$(x / c \approx 0.4)$, whilst the incompressible solver shows transition closer to the leading edge $(x / c \approx 0.2)$. We have observed significant variations of the transition location for the compressible solver when varying the polynomial order, that we have not seen in the incompressible solver. Further studies are necessary to completely assess the influence of discretisation in the transition location for the two solvers.

Second, we explore the pressure coefficient distribution along the airfoil profile when varying the Reynolds number. We only depict results for the incompressible DG solver since these are very similar to the results provided by the compressible solver. Note that this is not surprising, since the lift coefficients at $R e=1 \times 10^{5}$ and $R e=1 \times 10^{6}$ are very similar for $\mathrm{P}=4$ at $A o A=5^{\circ}$, see Table 1 . Figure 5 shows velocity contours for $R e=1 \times 10^{4}, R e=1 \times 10^{5}$ and $R e=1 \times 10^{6}$ at $A o A=5^{\circ}$. It can be seen that for the lowest Reynolds, the boundary layer remains laminar until it detaches after the maximum thickness, showing a highly unsteady wake. When the Reynolds number increases, the boundary layer shows transition to turbulence before the maximum thickness, as appreciated by the fluctuations and small scales appearing in Fig. 5.

To quantify these results, we depict in Fig. 6, the pressure distribution ( $\mathrm{Cp}$ ) for the three Reynolds numbers. In the top row, we show instantaneous $\mathrm{Cp}$ against averaged for incompressible DG solver. In the bottom row, we compare mean Cp distributions against Xfoil [34] (with critical N-factor $N_{c r}=1$ ) and Fluent SST (fully turbulent 

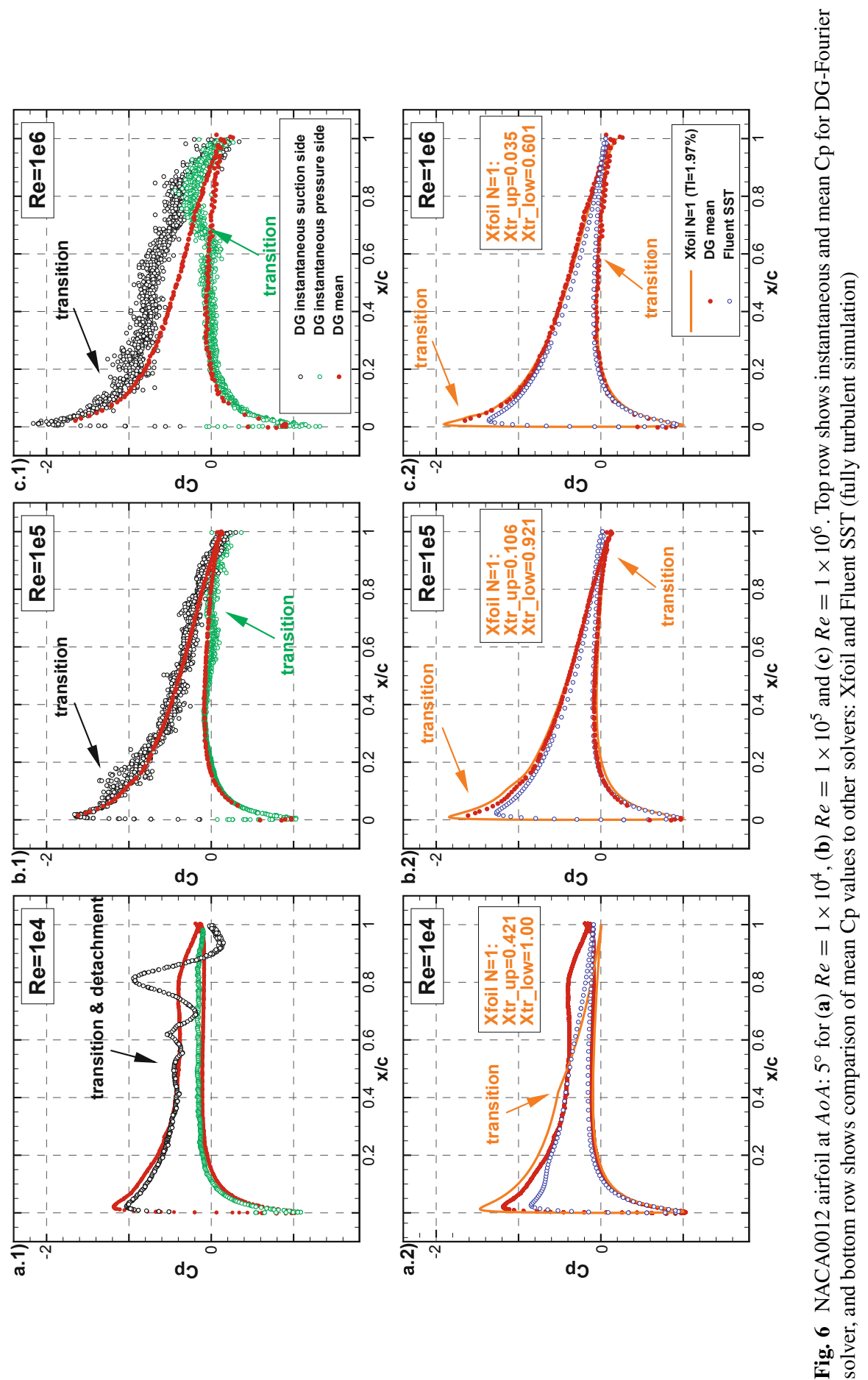
simulation) [35]. At $R e=1 \times 10^{4}$, the top figure shows that the boundary layer detaches before transition occurs and after the maximum thickness, as shown by the velocity contours in Fig. 5. Since the flow detaches leading to a highly unsteady wake, there is little hope that the averaged $\mathrm{Cp}$ captures the actual behaviour of the boundary layer. This is why, in the bottom figure, the mean values obtained using the incompressible DG solver do not agree with the mean Xfoil and Fluent values that assume steady turbulent flow. At $R e=1 \times 10^{5}$ and At $R e=1 \times 10^{6}$, the instantaneous $\mathrm{Cp}$ values (top row) show scattering in the data associated to transition. This occurs close to the leading edge on the suction side, whilst it is delayed towards the trailing edge on the pressure side. The bottom row shows that the DG results compare very well to Xfoil when using a critical $\mathrm{N}=1$ (to set the transition point close to the leading edge), whilst Fluent SST (fully turbulent) shows lower $\mathrm{Cp}$ values associated to simulating the complete boundary layer as turbulent (no laminar region). This results suggest that DG solvers using iLES approaches (compressible and incompressible) can capture transitional behaviour in boundary layers even when relatively coarse meshes are selected.

\section{Conclusions}

In this contribution, we have presented results for turbulent flows over a NACA0012 airfoil. High order discontinuous Galerkin formulations require localised dissipation to remain stable for under-resolved turbulent flow conditions, often referred to as implicit Large Eddy Simulations. Here we have presented compressible and an incompressible DG formulations (with different stabilising mechanisms) that are able to cope with high Reynolds number flows. Both DG formulations provide aerodynamic coefficients and boundary layer information that compare favorably to experimental data and well established low order solvers. We conclude that the compressible and incompressible formulations included in this work can be very useful in aeronautical applications.

Acknowledgements This project has received funding from the European Union Horizon 2020 Research and Innovation Program under the Marie Skłodowska-Curie grant agreement No 675008 for the SSeMID project. The authors acknowledge the computer resources and technical assistance provided by the Centro de Supercomputación y Visualización de Madrid (CeSViMa).

\section{References}

1. Hesthaven, J.S., Warburton, T.: Nodal Discontinuous Galerkin Methods - Algorithms, Analysis, and Applications. Springer, Berlin (2008)

2. Moura, R.C., Sherwin, S.J., Peiro, J.: Eigensolution analysis of spectral/hp continuous Galerkin approximations to advection-diffusion problems: insights into spectral vanishing viscosity. J. Comput. Phys. 307, 401-422 (2016) 
3. Gassner, G.J., Beck, A.D.: On the accuracy of high-order discretizations for underresolved turbulence simulations. Theor. Comput. Fluid Dyn. 27(3-4), 221-237 (2013)

4. Sherwin, S.: Dispersion analysis of the continuous and discontinuous Galerkin formulations. In: International Symposium on Discontinuous Galerkin Methods, pp. 425-431. Springer, Berlin (1999)

5. Manzanero, J., Rubio, G., Ferrer, E., Valero, E.: Dispersion-dissipation analysis for advection problems with nonconstant coefficients: applications to discontinuous Galerkin formulations. SIAM J. Sci. Comput. 40(2), A747-A768 (2018)

6. Canuto, C., Hussaini, M.Y., Quarteroni, A., Zang, T.A.: Spectral Methods in Fluid Dynamics. Springer, Berlin (1988)

7. Zang, T.A.: On the rotation and skew-symmetric forms for incompressible flow simulations. Appl. Numer. Math. 7(1), 27-40 (1991)

8. Blaisdell, G.A., Spyropoulos, E.T., Qin, J.H.: The effect of the formulation of nonlinear terms on aliasing errors in spectral methods. Appl. Numer. Math. 21(3), 207-219 (1996)

9. Ferrer, E.: An interior penalty stabilised incompressible discontinuous Galerkin-Fourier solver for implicit large eddy simulations. J. Comput. Phys. 348, 754-775 (2017)

10. Kirby, R.M., Sherwin, S.J.: Aliasing errors due to quadratic non-linearities on triangular spectral h/p element discretisations. J. Eng. Math. 56(3), 273-288 (2006)

11. Kirby, R.M., Karniadakis, G.E.: De-aliasing on non-uniform grids: algorithms and applications. J. Comput. Phys. 191, 249-264 (2003)

12. Mengaldo, G., De Grazia, D., Moxey, D., Vincent, P.E., Sherwin, S.J.: Dealiasing techniques for high-order spectral element methods on regular and irregular grids. J. Comput. Phys. 299, 56-81 (2015)

13. Blackburn, H.M., Schmidt, S.: Spectral element filtering techniques for large eddy simulation with dynamic estimation. J. Comput. Phys. 186(2), 610-629 (2003)

14. Rubio, G., Valero, E., Manzanero, J., Ferrer, E.: On the role of numerical dissipation in stabilising under-resolved turbulent simulations using discontinuous Galerkin methods (2018). arXiv:1805.10519

15. Arnold, D.N., Brezzi, F., Cockburn, B., Marini, L.D.: Unified analysis of discontinuous Galerkin methods for elliptic problems. SIAM J. Numer. Anal. 39(5), 1749-1779 (2001)

16. Wang, Z.J., Fidkowski, K., Abgrall, R., Bassi, F., Caraeni, D., Cary, A., Deconinck, H., Hartmann, R., Hillewaert, K., Huynh, H.T., Kroll, N., May, G., Persson, P.O., van Leer, B., Visbal, M.: High-order CFD methods: current status and perspective. Int. J. Numer. Methods Fluids 72(8), 811-845 (2013)

17. Beck, A.D., Bolemann, T., Flad, D., Frank, H., Gassner, G.J., Hindenlang, F., Munz, C.D.: High-order discontinuous Galerkin spectral element methods for transitional and turbulent flow simulations. Int. J. Numer. Methods Fluids 76(8), 522-548 (2014)

18. Flad, D., Gassner, G.: On the use of kinetic energy preserving DG-schemes for large Eddy simulation. J. Comput. Phys. 350, 782-795 (2017)

19. Kopriva, D.A.: Implementing Spectral Methods for Partial Differential Equations. Springer Netherlands, Dordrecht (2009)

20. Gassner, G.J., Winters, A.R., Kopriva, D.A.:. Split form nodal discontinuous Galerkin schemes with Summation-By-Parts property for the compressible Euler equations. J. Comput. Phys. 327, 39-66 (2016)

21. Gassner, G.J., Beck, A.D.: On the accuracy of high-order discretizations for underresolved turbulence simulations. Theor. Comput. Fluid Dyn. 27, 221 (2013)

22. Kopriva, D.A., Gassner, G.J.: An energy stable discontinuous Galerkin spectral element discretization for variable coefficient advection problems. SIAM J. Sci. Comput. 36(4), A2076-A2099 (2014)

23. Manzanero, J., Rueda-Ramirez, A.M., Rubio, G., Ferrer, E.: The Bassi Rebay 1 scheme is a special case of the symmetric interior penalty formulation for discontinuous Galerkin discretisations with Gauss-Lobatto points. J. Comput. Phys. 363, 1-10 (2018)

24. Gassner, G.J., Winters, A.R., Hindenlang, F.J., Kopriva, D.A.: The BR1 scheme is stable for the compressible Navier-Stokes equations. J. Sci. Comput. 77, 154 (2018) 
25. Ferrer, E., Willden, R.H.J.: A high order discontinuous Galerkin - Fourier incompressible 3D Navier-Stokes solver with rotating sliding meshes. J. Comput. Phys. 231(21), 7037-7056 (2012)

26. Ferrer, E.: A high order discontinuous Galerkin - Fourier incompressible 3D Navier-Stokes solver with rotating sliding meshes for simulating cross-flow turbines. PhD thesis, University of Oxford (2012)

27. Ferrer, E., Willden, R.H.J.: A high order discontinuous Galerkin finite element solver for the incompressible Navier-Stokes equations. Comput. Fluids 46(1), 224-230 (2011)

28. Ferrer, E., Moxey, D., Willden, R.H.J., Sherwin, S.: Stability of projection methods for incompressible flows using high order pressure-velocity pairs of same degree: continuous and discontinuous Galerkin formulations. Commun. Comput. Phys. 16(3), 817-840 (2014)

29. Le Clainche, S., Ferrer, E.: A reduced order model to predict transient flows around straight bladed vertical axis wind turbines. Energies 11(3), 566 (2018)

30. Ferrer, E., de Vicente, J., Valero, E.: Low cost 3D global instability analysis and flow sensitivity based on dynamic mode decomposition and high-order numerical tools. Int. J. Numer. Methods Fluids 76(3), 169-184 (2014)

31. Ladson, C.L., United States National Aeronautics and Space Administration, Scientific and Technical Information Division: Effects of independent variation of Mach and Reynolds numbers on the low-speed aerodynamic characteristics of the NACA 0012 airfoil section. NASA Technical Memorandum. National Aeronautics and Space Administration, Scientific and Technical Information Division (1988)

32. Gregory, N., O'Reilly, C.L., H.M.S.O.: Low-speed aerodynamic characteristics of NACA 0012 aerofoil section, including the effects of upper-surface roughness simulating hoar frost. Aeronautical Res. Council. Reports and Memoranda. National Physical Laboratory (1970)

33. Abbott, I.H.A., Von Doenhoff, A.E.: Theory of Wing Sections, Including a Summary of Airfoil Data. Dover Books on Engineering. Dover Publications, New York (1959)

34. Drela, M.: Xfoil: an analysis and design system for low Reynolds number airfoils. In: Mueller, T.J. (ed.) Low Reynolds Number Aerodynamics, pp. 1-12. Springer, Berlin (1989)

35. Fluent-ANSYS Academic Research Mechanical, Release 18.1, ANSYS Inc., Canonsburg (2017)

Open Access This chapter is licensed under the terms of the Creative Commons Attribution 4.0 International License (http://creativecommons.org/licenses/by/4.0/), which permits use, sharing, adaptation, distribution and reproduction in any medium or format, as long as you give appropriate credit to the original author(s) and the source, provide a link to the Creative Commons licence and indicate if changes were made.

The images or other third party material in this chapter are included in the chapter's Creative Commons licence, unless indicated otherwise in a credit line to the material. If material is not included in the chapter's Creative Commons licence and your intended use is not permitted by statutory regulation or exceeds the permitted use, you will need to obtain permission directly from the copyright holder.

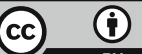

